Mens

Revue d'histoire intellectuelle et culturelle

mens

\title{
L'éphémère consécration de Juana, mon aimée, roman de Harry Bernard
}

\section{Guy Gaudreau et Micheline Tremblay}

Volume 15, numéro 2, printemps 2015

URI : https://id.erudit.org/iderudit/1036183ar

DOI : https://doi.org/10.7202/1036183ar

Aller au sommaire du numéro

Éditeur(s)

Centre de recherche en civilisation canadienne-française

ISSN

1492-8647 (imprimé)

1927-9299 (numérique)

Découvrir la revue

Citer cet article

Gaudreau, G. \& Tremblay, M. (2015). L'éphémère consécration de Juana, mon aimée, roman de Harry Bernard. Mens, 15(2), 49-83.

https://doi.org/10.7202/1036183ar
Résumé de l'article

Cet article retrace la trajectoire du roman Juana, mon aimée de Harry Bernard, de sa première version parue à la fin de l'automne 1930 jusqu'à sa consécration concrétisée par l'obtention d'un prix David en septembre 1932. Cette consécration fut néanmoins bien éphémère puisque l'oeuvre est aujourd'hui absente de l'histoire littéraire. Grâce à une riche correspondance, conservée en grande partie dans le Fonds Harry-Bernard, et au dépouillement minutieux des divers comptes rendus, recensions et articles publiés dans les journaux et revues, l'article analyse le rôle de l'auteur et met en lumière les contributions décisives d'Alfred DesRochers, de Claude-Henri Grignon et d'Albert Pelletier dans le sort réservé au roman. L'examen de la réception critique permet également de montrer l'importance des débats privés, des réseaux d'amitié et des discussions de salon tout autant que le poids inégal des recensions dans la trajectoire de l'ouvrage. Il appert que deux discours dominants ont été tenus à propos de l'oeuvre : le premier, favorable, de Grignon, qui conduisit au prix David, et le second, très sévère, de Pelletier, qui a contribué, selon nous, à la faire sombrer dans l'oubli.
Ce document est protégé par la loi sur le droit d'auteur. L'utilisation des services d’Érudit (y compris la reproduction) est assujettie à sa politique d'utilisation que vous pouvez consulter en ligne.

https://apropos.erudit.org/fr/usagers/politique-dutilisation/ 


\title{
L'éphémère consécration de Juana, mon aimée, roman de Harry Bernard
}

\author{
Guy Gaudreau \\ Micheline Tremblay \\ Université Laurentienne
}

\section{Résumé}

Cet article retrace la trajectoire du roman Juana, mon aimée de Harry Bernard, de sa première version parue à la fin de l'automne 1930 jusqu'à sa consécration concrétisée par l'obtention d'un prix David en septembre 1932. Cette consécration fut néanmoins bien éphémère puisque l'œuvre est aujourd'hui absente de l'histoire littéraire. Grâce à une riche correspondance, conservée en grande partie dans le Fonds Harry-Bernard, et au dépouillement minutieux des divers comptes rendus, recensions et articles publiés dans les journaux et revues, l'article analyse le rôle de l'auteur et met en lumière les contributions décisives d'Alfred DesRochers, de Claude-Henri Grignon et d'Albert Pelletier dans le sort réservé au roman. L'examen de la réception critique permet également de montrer l'importance des débats privés, des réseaux d'amitié et des discussions de salon tout autant que le poids inégal des recensions dans la trajectoire de l'ouvrage. Il appert que deux discours dominants ont été tenus à propos de l'œuvre : le premier, favorable, de Grignon, qui conduisit au prix David, et le second, très sévère, de Pelletier, qui a contribué, selon nous, à la faire sombrer dans l'oubli.

\section{Abstract}

This article retraces the story of Juana, mon aimée, by Harry Bernard, from its first draft, in the late autumn of 1930, to its triumph in Septem- 
ber of 1932, when the novel received the prix David. The book's success was short-lived, however, since it is now largely absent from Quebec's literary history. An examination of the extensive correspondence contained in the Harry-Bernard Fonds and of the various reviews of Juana, mon aimée that were published in the periodic press reveals the important role of the author and of Alfred DesRochers, Claude-Henri Grignon and Albert Pelletier in the novel's destiny. The book's critical reception shows that private debates, friendship, and drawing room discussions were as important to this destiny as were literary reviews. It would appear that two opinions dominated the critical reception: the first, which was formulated by Grignon, led to the prix David, while the second, which was formulated by Pelletier, led to the book's eventual descent into oblivion.

Le 4 octobre 1932, Athanase David, secrétaire de la province de Québec, fait parvenir à Harry Bernard ce mot : «Il me fait plaisir de vous inclure le chèque portant le numéro B21416, fait à votre ordre, pour le montant de $1,700 \$$, représentant le prix qui vous a été adjugé par le jury chargé de juger les œuvres littéraires ${ }^{1}$. $C^{\prime}$ 'est ainsi qu'au plus fort de la crise économique, le romancier reçut pour son roman Juana, mon aimée l'équivalent en dollars d'aujourd'hui de près de $30000 \$$, dans le cadre du concours des prix David que le Gouvernement du Québec avait institué en $1923^{2}$.

Deux semaines auparavant, on avait révélé le nom des cinq lauréats des prix David lors d'une cérémonie tenue à Montréal afin de récompenser les œuvres de fiction ${ }^{3}$. Respectant la séquence triennale

1 Athanase David à Harry Bernard, 4 octobre 1932, Bibliothèque et Archives nationales du Québec Vieux-Montréal (ci-après BAnQ-VM), Fonds Harry-Bernard (ci-après Fonds HB), MSS298/011/002.

2 Silvie Bernier, Prix littéraires et champs du pouvoir : le prix David, 1923-1970, mémoire de maîtrise (études françaises), Sherbrooke, Université de Sherbrooke, 1983; pour l'origine et le contexte historique entourant la création du prix, voir le chapitre deux, p. 22-47.

3 Les quatre autres gagnants étaient Alfred DesRochers, pour son recueil $\grave{A}$ l'ombre de l'Orford, qui avait partagé avec Robert Choquette et son Metropolitan Museum 
des concours établie depuis quelques années, celui de 1932 avait porté uniquement sur les œuvres publiées au cours des trois années précédentes ${ }^{4}$. Afin de multiplier ses chances, Bernard avait soumis également un autre roman, La ferme des pins, publié en 1930 chez le même éditeur, Albert Lévesque 5 .

Ce succès de Juana, mon aimée, roman absent des ouvrages récents d'histoire littéraire ${ }^{6}$, mérite qu'on s'y arrête. Quelles sont les étapes qui ont mené à cette consécration, quel rôle actif a joué Bernard et pourquoi cet effacement aujourd'hui? L'histoire de ce roman, riche d'enseignement, permet d'éclairer l'institution littéraire canadiennefrançaise qui se consolide à cette époque alors que s'installe l'âge de la critique. Parallèlement, cet article poursuit notre recherche ${ }^{7}$ lancée

le prix de poésie, chacun touchant 850 \$. Un prix de 600 \$ avait également été accordé à Madame Lorenzo Montreuil pour son roman en langue anglaise Dumbel, et un dernier de même valeur à Gertrude Bartlett pour son recueil de poésie The White Bird (voir "Le résultat du concours annuel du prix David ", Le Soleil, 20 septembre 1932, p. 1).

4 "Historique des prix du Québec ", [s. d.], sur le site Web du Gouvernement du Québec, [http://www.prixduquebec.gouv.qc.ca/publications/historique.pdf] (10 janvier 2015).

5 Harry Bernard à Albert Lévesque, 14 janvier 1932, BAnQ-VM, Fonds HB, MSS298/046/014.

${ }^{6}$ L'ouvrage, sous la direction de Denis Saint-Jacques et Lucie Robert, est à ce titre fort éloquent, car le roman n'est même pas cité dans l'index des œuvres (La vie littéraire au Québec, t. VI : 1919-1933 : le nationaliste, l'individualiste et le marchand, Québec, Les Presses de l'Université Laval, 2010, p. 725).

7 Guy Gaudreau et Micheline Tremblay, « Harry Bernard (1898-1979) : érudit et homme de lettres ", Mens : revue d'histoire intellectuelle de l'Amérique française, vol. 2, no 1 (automne 2001), p. 35-65; Micheline Tremblay et Guy Gaudreau, "Le régionalisme littéraire au Canada français : le point de vue de Harry Bernard ", Globe : revue internationale d'études québécoises, vol. 5, n 1 (2002), p. 159-178; Guy Gaudreau et Micheline Tremblay, "Harry Bernard, journaliste au Droit, 1919-1923", Revue du Nouvel-Ontario, n 28 (2003), p. 51-77; Harry Bernard, Conversation poétique : correspondance littéraire entre Harry Bernard et Alfred DesRochers, textes établis, présentés et annotés par Micheline Tremblay et Guy Gaudreau, Ottawa, Éditions David, 2005 (ci-après Conversation poétique); Simone Routier, Je voudrais bien être un homme : correspondance littéraire entre Simone Routier et Harry Bernard, textes établis, présentés et annotés par Guy Gaudreau et Micheline Tremblay, Ottawa, Éditions David, 2011; Harry Bernard, Une autre 
il y a près de vingt ans sur ce Maskoutain oublié depuis la Révolution tranquille et qui fut pourtant, à son époque, reconnu comme un des bons romanciers du Canada français en remportant un prix David à trois reprises.

Pour nourrir cette réflexion, nous avons puisé à même la riche correspondance de Bernard - conservée, notamment, dans son fonds d'archives à Bibliothèque et Archives nationales du Québec - qui révèle les étapes antérieures à la publication du roman et les confidences d'écrivains dans la sphère privée. Pour ce qui est de la sphère publique et de l'accueil critique réservé à l'œuvre, nous avons dépouillé une trentaine de publications et recueilli une cinquantaine de comptes rendus et d'articles, qui exposent un intéressant système de réception ${ }^{8}$ et mettent en évidence les opinions des principaux ténors de l'époque. Ces commentaires, largement positifs, ont sûrement influencé l'évaluation des sept membres francophones du jury ${ }^{9}$. Numérisés et accessibles en ligne en version intégrale ${ }^{10}$, ces textes présentent un large éventail

année sera meilleure, édition préparée par Micheline Tremblay et Guy Gaudreau, Ottawa, Éditions David, 2013; Guy Gaudreau et Micheline Tremblay, «Technique de mise en pages, crise économique et journalisme d'opinion : Le Courrier de SaintHyacinthe de 1920 à 1938 ", Scientia Canadensis: Canadian Journal of the History of Science, Technology and Medecine = Revue canadienne d'histoire des sciences, des techniques et de la médecine, vol. 36, n 2 (2013), p. 37-62. On trouvera une version numérique des quatre articles et des textes de présentation des ouvrages cités sur le site Web Les écrits de Harry Bernard, [http://harry-bernard.cegepmontpetit.ca/ travaux-sur-harry-bernard] (20 mai 2015).

8 Voir Daniel Chartier, L'émergence des classiques : la réception de la littérature québécoise des années 1930, Montréal, Éditions Fides, 2000.

9 Ces membres seront : Jean-Charles Harvey, Louvigny de Montigny, Ægidius Fauteux, Louis-Philippe Geoffrion, Léon Lorrain, les abbés Olivier Maurault et Aimé Labrie (voir Bernier, Prix littéraires et champs du pouvoir, p. 146; et pour une analyse de l'origine des jurys au fil du temps, voir le chapitre 4 de son mémoire, p. 69-93). Quant à la question de la composition des membres du jury dans la sélection des lauréats d'un prix littéraire, on pourra lire l'article de Richard Giguère, "L'offrande aux vierges folles », dans Pierre Hébert, Kenneth Landry et Yves Lever (dir.), Dictionnaire de la censure au Québec : littérature et cinéma, Montréal, Éditions Fides, 2006, p. 490-497; voir, plus particulièrement, p. 495.

${ }^{10}$ La mise en ligne de tous ces comptes rendus permet aux lecteurs un accès direct au texte et nous autorise du même souffle à prendre une certaine liberté dans la 
de points de vue et permettent de prendre la mesure des qualités et des défauts retenus par les critiques.

\section{Juana, mon aimée dans l'œuvre de Harry Bernard}

D'abord quelques mots sur l'auteur. Harry Bernard ${ }^{11}$ naquit à Londres en 1898, où son père Horace, représentant commercial d'un importateur de foin, s'était installé avec la famille pour quelque temps. Après un séjour à Paris (où il entreprend son cours primaire) et un déménagement aux États-Unis, sa famille s'installe à SaintHyacinthe, juste à temps pour qu'il commence son cours classique au Séminaire, cours qu'il terminera en 1919. Pendant ses études, il fait ses premières armes de journaliste au Courrier de Saint-Hyacinthe. Après l'obtention de son baccalauréat, il décide de faire carrière en journalisme et il apprend son métier au quotidien Le Droit d'Ottawa. En 1923, il décroche le poste de rédacteur en chef du Courrier, poste qu'il occupera jusqu’à sa retraite, en 1970.

Comme pour bien d'autres auteurs de cette époque, le journalisme lui sert de gagne-pain. En 1922, il peut ainsi commencer un premier roman, L'homme tombét ${ }^{2}$, qu'il soumet, pour commentaires, à Lionel Groulx avec qui il va collaborer régulièrement à L'Action française ${ }^{13}$.

transcription des titres et des extraits cités, évitant, par exemple, l'utilisation du [sic] et une variation dans l'identification de certains intitulés, voir le site Web Les écrits de Harry Bernard, [http://harry-bernard.com/reception-critique-desoeuvres-de-harry-bernard/juana-mon-aimee] (20 janvier 2016).

11 Pour sa biographie, on consultera notre article paru dans la revue Mens en 2001, mais également une chronologie plus étoffée, nourrie des recherches menées depuis, sur le site Web Les écrits de Harry Bernard, [harry-bernard.com]. En outre, on pourra y lire ses mémoires, inédits jusqu'à leur mise en ligne en 2014.

12 Mentionnons que c'est à la lecture de ce roman que l'idée de s'intéresser à Harry Bernard nous est venue il y a plus de 20 ans. L'homme tombé est un des premiers romans à décrire l'influence néfaste du cinéma sur un des personnages (voir Micheline Tremblay, La présence du cinéma dans le roman canadien-français de 1896 à 1970, Université Paul-Valéry, Montpellier III, 1994).

13 Cette collaboration de Bernard avec Groulx a été relatée dans Jacques Michon (dir.), Histoire de l'édition littéraire au Québec au XX' siècle, vol. I : La naissance de l'éditeur : 1900-1939, Montréal, Éditions Fides, 1999, p. 259-261. 
Publié l'année suivante, le roman s'inscrit dans le courant régionaliste, qui s'impose comme le courant littéraire dominant à l'époque ${ }^{14}$. Par la suite, paraissent d'autres romans au service de la nation dans lesquels, pour reprendre l'expression de Lucie Robert, Bernard fait œuvre nationale plutôt qu'œuvre littéraire ${ }^{15}$. Il s'y emploie avec assiduité, engrangeant presque une œuvre par année. À deux reprises, il décroche un prix David, un pour L’homme tombé en 1924 (500\$) et un autre pour La terre vivante en $1926(500 \$)^{16}$. Compte tenu de leur faible qualité littéraire, c'est assurément la thèse plus que le contenu qu'on récompense alors. Dans toute son écriture romanesque, il conservera un point de vue régionaliste en prônant un vocabulaire de chez nous, une description des lieux, de la faune et de la flore qui nous représentent. Tel est le romancier encore à l'œuvre dans La ferme des pins publié en 1930 et soumis à ce concours de 1932.

Mais Juana, mon aimée est d'une tout autre facture. Ayant besoin de repos, le journaliste Raymond Chatel s'exile en Saskatchewan où il rencontre Juana. Suite à un malentendu - que les critiques jugeront boiteux et fort discutable -, ils devront mettre fin à leur idylle. Si ce roman a beaucoup plu à sa sortie, c'est qu'il en a surpris plusieurs, en évacuant le roman à thèse au profit d'une histoire d'amour et en adoptant une structure narrative peu connue au Canada français, qui abandonne le narrateur omniscient.

${ }^{14}$ Sur cette question, on lira l'incontournable ouvrage d'Annette Hayward, La querelle du régionalisme au Québec (1904-1931) : vers l'autonomisation de la littérature québécoise, Ottawa, Le Nordir, 2006.

15 Lucie Robert, "L'institution littéraire ", dans Denise Lemieux (dir.), Traité de la culture, Québec, Éditions de l'Institut québécois de recherche sur la culture (IQRC), 2002, p. 352.

16 Pour un résumé et une analyse de ces romans de la première heure, voir les deux articles de Jean-Paul Lamy dans Maurice Lemire (dir.), Dictionnaire des auvres littéraires du Québec, t. II : 1900 à 1939, Montréal, Éditions Fides, 1980, "L'homme tombé, roman de Harry Bernard ", p. 573-574, et "La terre vivante et autres romans de la terre de Harry Bernard », p. 1068-1071. 


\section{La rédaction et l'impression de la version définitive}

Fidèle à ses habitudes, Bernard soumet d'abord le manuscrit de Juana à Groulx en décembre 1930. Au début janvier, Groulx le félicite de ses progrès : "Quand je me rappelle votre laborieuse manière des premiers temps, je trouve franchement à me réjouir et admirer, devant ce style alerte, aéré, fleur de soleil ${ }^{17}$. " Terroiriste et adepte d'une littérature utile à la nation, le chanoine lui avoue, toutefois, sa préférence pour La terre vivante.

Comme il l'avait fait pour La ferme des pins ${ }^{18}$, Bernard demande également l'avis de son ami Alfred DesRochers qui, le 8 janvier $1931^{19}$, l'invite une première fois à lui soumettre le manuscrit, puis à nouveau le $23^{20}$. Bernard, qui admire la poésie de DesRochers, a confiance en lui; c'est un ami et un confident. C'est la raison pour laquelle il a accepté de faire partie de ce regroupement d'auteurs des Cantons de l'Est qu'anime DesRochers, appelé Les Écrivains de l'Est. Le poète de Sherbrooke jouera, comme nous le verrons plus loin, un rôle clé dans le succès de Juana. Finalement, le 26 janvier, Bernard lui envoie le manuscrit. "Relativement à Juana, je te demande de me signaler toutes les fautes de français et de goût, les impropriétés, les anachronismes, les sottises de toutes variétés que tu pourras y trouver ${ }^{21}$."

Dès le lendemain, DesRochers lui adresse quelques remarques fort pertinentes dans lesquelles il le met en garde contre le monologue intérieur, peu compris des lecteurs; il souligne aussi le manque d'émotion, de sensualité de ce texte pourtant axé sur le sentiment amoureux.

Et ça m'amène à ton roman, dont je viens de lire 22 pages sur le temps de la compagnie. C'est très bien jusque-là. Seulement, ici

17 Lionel Groulx à Harry Bernard, 2 janvier 1931, Fondation Lionel-Groulx, Fonds Lionel-Groulx, CLG1.

18 Alfred DesRochers à Harry Bernard (ci-après $\mathrm{AD}$ à $\mathrm{HB}$ ou HB à $\mathrm{AD}), 21$ avril 1930, Conversation poétique, p. 99.

$19 \mathrm{AD}$ à HB, 8 janvier 1931, Ibid., p. 161.

$20 \mathrm{AD}$ à HB, 23 janvier 1931, Ibid., p. 169.

${ }^{21} \mathrm{HB}$ à AD, 26 janvier 1931, Ibid., p. 173. 
encore, ce roman a trop de technique pour plaire à nos imbéciles. $\mathrm{Nul}$ ne comprend le procédé du monologue intérieur, l'une des plus belles découvertes de l'écriture moderne. Mon roman Nous avons joué dans l'Île est justement composé d'après ce procédé, ce qui me fait douter qu'il y ait une demi-douzaine de personnes qui le comprennent ${ }^{22}$.

Quant au style de Juana, il me semble supérieur à tout ce que tu as écrit à date - et c'est dans l'ordre. Il y a une chose qui m'étonne et je te le dis tout de suite, c'est que quand tu dépeins un intérieur ou un caractère, je ne trouve pas un mot à redire; mais dès que tu parles d'un paysage, il faut que tu le métaphysicises. J'ai noté au passage, "des silences complets, immenses, et poignants ». C'est peut-être parce que je suis essentiellement sensuel, mais ces mots-là ne me font rien voir. Pourquoi pas un silence «cru ». Ça me transirait et me ferait ressentir toutes les impressions que tu as transposées dans tes épithètes métaphysiques ${ }^{23}$.

Dès le début de février, lors d'une rencontre à Montréal, DesRochers informe Albert Lévesque du projet de Bernard. Sans avoir lu le manuscrit, l'éditeur ne semble pas, toutefois, enthousiasmé par le roman ${ }^{24}$.

Le 23 février, DesRochers retourne à Bernard le manuscrit annoté, accompagné d'une longue lettre dont nous citons quelques extraits ${ }^{25}$ :

Mon cher Bernard,

J'ai tenu ma promesse à Juana. J'ai passé toute une après-midi en sa compagnie et je me suis permis de faire des annotations dans les marges trop étroites du malcommode papier où elle est embaumée.

C'est ton roman le plus solidement étoffé encore. [...]

Ta phrase est trop volontairement dépouillée de tout ornement.

${ }^{22}$ Comme on le verra plus loin, la remarque de DesRochers sur la nouveauté du procédé narratif de Bernard est tout à fait juste.

$23 \mathrm{AD}$ à HB, 27 janvier 1931, Conversation poétique, p. 175.

${ }^{24} \mathrm{HB}$ à $\mathrm{AD}, 6$ février 1931, Ibid., p. 182.

25 AD à HB, 23 février 1931, Ibid., p. 198-200. 
Je sais aussi bien que toi que c'est la mode d'aujourd'hui; qu'aux États-Unis, Hemingway et ses suivants se font un devoir de tordre le cou à l'éloquence, mais - et tu ne me sembles pas l'avoir complètement évité - le danger de cette méthode, c'est que le tour de phrase devienne standardisé. Quand on a lu une dizaine de pages, on a tellement ta syntaxe en tête qu'on perd tout espoir d'imprévu.

$[\ldots]$

Tu viens de renouveler complètement ta pensée et tes domaines d'intérêt en abordant l'amour et la psychologie féminine, où tu fais des notations d'une finesse peu accoutumée au pays; renouvelle donc ton style, maintenant. Il ne s'agirait que de tourner différemment une phrase ici et là, de fondre ensemble trois ou quatre de tes phrases nerveuses en une période pulpeuse; de changer quelques épithètes de place et le tour serait joué.

$[\ldots]$

Bonjour, et dis-moi que tu te sacreras de mes remarques.

Alfred D.

Ayant probablement tenu compte, du moins en partie, des commentaires de DesRochers, Bernard lui soumet une nouvelle version le $1^{\text {er }}$ mars. "Naturellement ", comme il le mentionne ce jour-là, " je me réserve le droit de ne pas tenir compte de tes indications. De temps à autre, je ferai les corrections indiquées. Et je passerai pour bien écrire ma langue, grâce à toi ${ }^{26}$."

Ne disposant ni des versions soumises ni des manuscrits annotés, nous ne pouvons comparer avec la version imprimée et mesurer ainsi l'ampleur des corrections suggérées et l'importance de la contribution de DesRochers. Signalons que le 12 mai, Bernard avouera à son ami : "Quant à Juana, je ne l'ai pas revue. Elle aura grandi quand je reprendrai mes attentions, et elle ne sera que plus belle fille ${ }^{27}$. "Cela nous porte à croire qu'il a laissé dormir son manuscrit quelque temps.

${ }^{26} \mathrm{HB}$ à $\mathrm{AD}, 1^{\text {er }}$ mars 1931, Ibid., p. 204.

$27 \mathrm{HB}$ à $\mathrm{AD}, 12$ mai 1931, Ibid., p. 215. 
Lété 1931 demeure très chargé pour Bernard. La campagne électorale - les élections ont lieu le 24 août - ainsi que les deux poursuites judiciaires pour libelle diffamatoir ${ }^{28}$ dans lesquelles il est impliqué contre le député et maire de Saint-Hyacinthe T.-D. Bouchard l'accaparent sans doute beaucoup. Il réussit tout de même à soumettre une dernière version à Donatien Frémont le 16 juillet. "Je vous demanderais donc d'en prendre connaissance et de l'écheniller soigneusement au point de vue des erreurs, anachronismes, naïvetés touchant la vie de l'Ouest et de la Saskatchewan en particulier--, qui auraient pu s'y glisser ${ }^{29}$. » Moins de deux semaines plus tard, Frémont le félicite pour la justesse de son portrait de l'Ouest, tout en lui suggérant une douzaine de corrections ${ }^{30}$.

C'est à titre de gérant de l'atelier d'imprimerie du Courrier de Saint-Hyacinthe chargé de l'impression du roman ${ }^{31}$, qu'il s'active à compter du mois d'août. Le $5^{32}$, il fait parvenir à Albert Lévesque des échantillons des caractères d'imprimerie qu'il compte utiliser. Dans la même foulée, Bernard l'informe qu'il lui retournera prochainement le contrat d'édition du roman. Le 26, il fait connaitre son choix de papier, du «Eggshell, 19 X 30, subat. $60 \mathrm{lbs}^{33}$ », et

${ }^{28}$ Pour un aperçu de ces deux causes judiciaires, voir le site Web Les écrits de Harry Bernard, [http://harry-bernard.cegepmontpetit.ca/1931-1932-defenseur-du-livreet-membre-de-differentes-associations/] (20 mai 2015).

${ }^{29}$ HBà Donatien Frémont, 16 juillet 1931, BAnQ-VM, Fonds HB, MSS298/011/001.

30 Donatien Frémont à HB, 29 juillet 1931, BAnQ-VM, Fonds HB, MSS298/011/001. Claude Melançon est un autre ami qui a lu le manuscrit, sans que l'on sache cependant quand il l'a commenté (voir Claude Melançon à HB, non daté, mais vraisemblablement en octobre ou novembre 1931, BAnQ-VM, Fonds HB, MSS298/011/001).

31 Précisons que ce sont ces mêmes presses, sous la supervision étroite de Bernard, qui vont imprimer À l'ombre de l'Orford, recueil de poésie de DesRochers primé lors du concours des prix David cette année-là.

${ }^{32}$ HB à Albert Lévesque, 5 août 1931, BAnQ-VM, Fonds HB, MSS298/046/014.

${ }^{33}$ HB à Albert Lévesque, 26 août 1931, BAnQ-VM, Fonds HB, MSS298/044/005. Le choix du papier n'est pas aussi banal qu'il y parait. Dans une entrevue que Lévesque a accordée à cette époque, il évoque le problème du papier, dont le choix et les coûts posent de sérieuses difficultés (voir "Difficultés d'un éditeur ", L'Illustration, $1^{\text {er }}$ octobre 1932, p. 5 et 20). 
demande à Lévesque de lui en envoyer dès qu'il en aura trouvé. Ce n'est que le 10 septembre $^{34}$ que l'éditeur l'avise de l'envoi prochain du papier recherché. Finalement, le 4 octobre 1931, 1000 exemplaires de Juana sortent des presses du Courrier de Saint-Hyacinthe.

\section{La promotion du roman}

Lévesque et Bernard sont bien conscients qu'il faut faire connaître le roman avant même sa parution afin d'en faciliter la vente. Dès avril, le romancier a tenté, sans succès, de convaincre Roméo Leblanc, secrétaire de la Revue de l'Université d'Ottawa, de faire paraître un chapitre inédit de Juana ${ }^{35}$. Ayant accepté, malgré ses multiples occupations, de rédiger la recension d'un ouvrage de Séraphin Marion pour cette revue ${ }^{36}$, il avait pensé obtenir cette faveur en contrepartie.

Le 27 août, Lévesque propose de faire parvenir à Jean Bruchési, de La Revue moderne, un extrait de Juana qui correspondrait à une page de la revue ${ }^{37}$. Bernard choisit les premières pages, qui paraîtront dans le numéro d'octobre. Le même mois, sans consulter Bernard cette fois ${ }^{38}$, Lévesque fait paraître un autre extrait dans Mon Magazine, en publiant intégralement le chapitre quatre ${ }^{39}$.

Annoncé dans ces magazines comme un roman d'amour, Juana cherche à attirer une clientèle féminine qu'on dit, à l'époque, friande

${ }^{34}$ Albert Lévesque à HB, 10 septembre 1931, BAnQ-VM, Fonds HB, MSS298/044/005.

35 HB à Roméo Leblanc, 22 avril 1931, BAnQ-VM, Fonds HB, MSS298/046/014.

36 Il s'agit de son ouvrage En feuilletant nos écrivains; Roméo Leblanc à $\mathrm{HB}$, 20 avril 1931, BAnQ-VM, Fonds HB, MSS298/046/014.

37 Albert Lévesque à HB, 27 août 1931, BAnQ-VM, Fonds HB, MSS298/046/014. Cet extrait correspond aux pages 11 à 21 de l'édition originale.

38 Bernard, dans une lettre à Ernest-Jules Larivière, de Mon Magazine, datée du 15 octobre, lui fera part de sa frustration consécutive à l'initiative de Lévesque : " Mais je vous saurais gré, à l'avenir, de vous adresser à moi-même, quand vous aurez besoin d'un texte" (BAnQ-VM, Fonds HB, MSS298/011/001).

39 «Extrait Juana, mon aimée : un nouveau roman d'Harry Bernard ", Mon Magazine, octobre 1931, p. 28-29. 
de ce genre de publication. L'auteur étant reconnu comme un adepte $\mathrm{du}$ régionalisme, les deux revues situent brièvement le roman par rapport à son œuvre. Conséquence de la confusion provoquée par la narration au « JE », dans La Revue moderne, le texte de présentation non signé ${ }^{40}$ - qu'on peut vraisemblablement attribuer à Jean Bruchésiindique que Bernard a vécu dans l'Ouest canadien et qu'il s'est inspiré d'un épisode de sa vie là-bas. De fait, il n'y a séjourné qu'une semaine tout au plus ${ }^{41}$ et le récit est pure fiction ${ }^{42}$.

Toujours dans le but de mousser les ventes, Lévesque fait paraitre, dans Le Terroir de Québec ${ }^{43}$, un communiqué non signé, mais probablement de la plume de Bernard, en même temps que le lancement officiel de Juana, soit entre le 18 et le 20 octobre. Ce communiqué sera repris intégralement dans Le Droit du 24 octobre, Le Courrier de Saint-Hyacinthe du 30 octobre et Le Devoir du 19 novembre, assurant à l'œuvre une large diffusion. Il y est question d'un roman qui « fait une plus large place à l'amour et à la psychologie

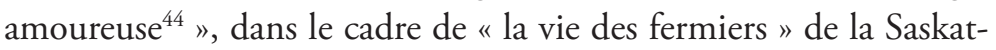
chewan. Et, afin de dissiper la confusion évoquée précédemment, le communiqué précise : « $\mathrm{M}$. Bernard a écrit son roman à la première personne. Il n'a pas craint de tenter ce genre, extrêmement difficile et fort en faveur chez les romanciers modernes, qu'on appelle le monologue intérieur, et qui offre tant de ressources à l'écrivain. »Un dernier communiqué, plus court celui-là, s'ajoute à l'appareil promo-

40 "Le roman inédit, Juana, mon aimée par Harry Bernard ", La Revue moderne, octobre 1931, p. 28.

${ }^{41}$ Ce bref séjour se déroula dans le cadre d'un voyage de la Liaison française à l'été 1927.

${ }^{42}$ La Revue moderne ne sera pas la seule à mal interpréter cette structure narrative; Georges-Émile Marquis fera de même, comme on peut le voir dans sa recension publiée dans Le Terroir en décembre 1931, p. 8.

43 Le choix d'un mensuel publié dans la région de Québec, après les deux publications montréalaises, s'explique peut-être par la volonté de Bernard ou de son éditeur de s'adresser à un autre bassin de lecteurs, d'autant plus que la démarche, qui avait échoué au printemps, visait un autre marché francophone, celui de la région outaouaise.

44 Le Terroir, octobre 1931, p. 29. 
tionnel le 23 octobre ${ }^{45}$, quelques jours après la parution du roman. Et dans cette même livraison du Courrier, Bernard insère un extrait du chapitre premier, à peine plus long que celui paru dans La Revue moderne ${ }^{46}$.

En tant que rédacteur en chef du Courrier, Bernard ne se gêne pas pour repiquer certaines critiques favorables, parues ailleurs, ou reproduire des articles de son cru d'abord publiés dans certaines revues connues. Son poste lui assure donc une tribune promotionnelle, et ce, en accord avec l'actionnaire majoritaire mais discret du journal, $\mathrm{M}^{\mathrm{gr}}$ Desranleau ${ }^{47}$. D'où la présence d'annonces publicitaires qui paraissent assez régulièrement dans $L e$ Courrier $^{48}$.

Si Lévesque doit se charger du service de presse, comme le prévoit le contrat d'édition ${ }^{49}$, c'est à Bernard de distribuer des exemplaires à ses amis et à des personnes qu'il n'a certes pas choisies au hasard. À la fin d'octobre, un exemplaire est posté à DesRochers et à Claude Melançon tandis qu’au début du mois suivant, un autre est destiné au critique Louis Dantin, grand ami et correspondant de DesRochers ${ }^{50}$.

45 "Un nouveau roman ", Le Courrier de Saint-Hyacinthe, 23 octobre 1931, p. 1.

46 Il s'agit des douze premières pages du roman, soit les pages 11 à 22.

47 On ignore à l'époque que $\mathrm{M}^{\mathrm{gr}} \mathrm{D}$ Desranleau est actionnaire majoritaire de l'entreprise qui édite le Courrier de Saint-Hyacinthe. Personne ne se doute des liens qui existent entre Bernard et Desranleau quand apparait, dans Le Devoir du 24 janvier 1925, la recension fort élogieuse de ce dernier au sujet de L'homme tombé. Cette recension sera d'ailleurs reproduite non seulement dans L'Action catholique, mais aussi dans Le Courrier (voir le site Web Les écrits de Harry Bernard, [http://harry-bernard. cegepmontpetit.ca/reception-critique-des-oeuvres-de-harry-bernard/receptioncritique-du-roman-lhomme-tombe/] (20 mai 2015)).

48 Les romans antérieurs de Bernard, comme Dolorès publié en 1932, eurent sensible ment droit au même traitement promotionnel dans Le Courrier.

49 Nous n'avons pu trouver ce contrat d'édition, mais tout porte à croire qu'il est très semblable à celui signé par DesRochers à la même époque et qui a été décrit par Richard Giguère (voir "Alfred DesRochers et ses éditeurs : des relations d'affaires tendues ", dans Jacques Michon (dir.), L'édition littéraire en quête d'autonomie : Albert Lévesque et son temps, Québec, Les Presses de l'Université Laval, 1994, p. 16-17).

50 Voir Pierre Hébert, Patricia Godbout et Richard Giguère (dir.), La correspondance entre Louis Dantin et Alfred DesRochers : une émulation littéraire (1928-1939), avec la collaboration de Stéphanie Bernier, Montréal, Éditions Fides, 2014 (ci-après Émulation littéraire). 
Quelques mots de remerciement envoyés à Bernard pour son envoi de Juana permettent de connaître les noms d'autres destinataires, dont certains sont très influents dans le monde littéraire. Outre les abbés Labrie et Maurault, qui feront partie des membres du jury du prix David, il y a aussi Maurice Hébert, le dominicain Marc-Antonin Lamarche, le chanoine Émile Chartier, Jovette Bernier et Léo-Paul Desrosiers.

\section{Les premiers comptes rendus}

La toute première recension parait le 29 octobre dans l'hebdomadaire La Revue de Granby. Son rédacteur en chef, Édouard Hains, qui ne prétend pas être critique littéraire, signe le texte ${ }^{51}$. Le premier intérêt de ce compte rendu est sans doute de confirmer l'existence d'un service de presse : les "volumes qu'Albert Lévesque nous adresse d'ailleurs avec une constance vraiment fraternelle ${ }^{52}$ ». Plus loin, Hains évoque explicitement le communiqué de l'éditeur, qui aurait ainsi rejoint un bon nombre de journaux et de revues. Si Hains fait état d'invraisemblances dans le récit, sa critique, loin d'être bâclée, demeure dans l'ensemble positive.

Le lendemain, Jean-Charles Harvey, romancier, journaliste et critique respecté du milieu littéraire, entre dans la danse en soulignant les forces et les faiblesses de Juana dans Le Soleil ${ }^{53}$. Quoique la pauvreté du style et la méconnaissance de la langue française y soient évoquées, Harvey souligne le progrès de l'auteur, qui s'est débarrassé de son ton apostolique, et conclut que le roman demeure, en somme, agréable.

Quelques jours plus tard, Bernard reçoit une lettre fort élogieuse de Dantin : «Votre Juana m'a plu extrêmement et m'a fait l'impression d'être, à tous points de vue, le meilleur des ouvrages que vous ayez

51 Quelques jours après la sortie du roman, Hains prévient Bernard qu'il publiera sous peu une critique (Édouard Hains à HB, 22 octobre 1931, BAnQ-VM, Fonds HB, MSS298/011/001).

52 Édouard Hains, "Le dernier de Harry Bernard : Juana, mon aimée ", La Revue de Granby, 29 octobre 1931, p. 3.

53 Jean-Charles Harvey, "Juana, mon aimée, roman canadien de M. Harry Bernard ", Le Soleil, 30 octobre 1931. 
écrits ${ }^{54}$. "Quoiqu'elle ait été émise privément, cette opinion du "critique le plus influent et le plus novateur de l'entre-deux-guerres 55 " rassure certainement Bernard sur la valeur de son roman et l'incite à reproduire la recension de Harvey dans Le Courrier ${ }^{56}$, en l'amputant toutefois des derniers paragraphes où il se montrait très sévère, comme on le constate dans cet extrait supprimé :

Qu'on n'y cherche toutefois rien de bien profond, rien de bien poignant. Harry Bernard reste à la surface des choses. Sa psychologie reste à la surface des choses. Sa psychologie est en superficie, médiocre en étendue d'ailleurs. [...]

Il semble que ce sont surtout les moyens d'expression qui manquent à l'écrivain. Celui-ci sait mieux construire un roman qu'une phrase. Là est sa faiblesse. Chez lui, jamais rien de pathétique, pas de frémissement, pas de nerfs. Son style court, haché qu'on ne lit que par respiration saccadée, nous essouffle sans nous soulever. [...]

Je me figure aisément que pour cet écrivain sympathique, le français est une langue apprise, c'est-à-dire une langue acquise par étude tardive plutôt que par l'éducation première. Je m'explique mieux par là la course laborieuse et la composition illogique de maintes phrases ainsi que le sens curieux donné à certains vocables.

Les trois recensions suivantes demeurent dans l'ensemble positives : malgré ses défauts, l'œuvre reste plaisante. Dans Mon Magazine $^{57}$, Jules-Ernest Larivière, s'il remet en question le traitement

${ }^{54}$ Louis Dantin à HB, 3 novembre 1931, BAnQ-VM, Fonds HB, MSS298/011/002.

55 Pierre Hébert, "La censure cléricale et la critique littéraire laïque au Québec ", dans Carole Gerson et Jacques Michon (dir.), Histoire du livre et de l'imprimé au Canada, vol. III : De 1918 à 1980, Montréal, Les Presses de l'Université de Montréal, 2007, p. 504.

56 Pour s'en convaincre, on peut lire également les deux versions de ce compte rendu : Jean-Charles Harvey, "Bibliographie canadienne : Juana, mon aimée, roman canadien de M. Harry Bernard ", Le Soleil, 30 octobre 1931, p. 4, et "L'une des plus charmantes fictions de ce pays ", Le Courrier de Saint-Hyacinthe, 6 novembre 1931, p. 1, sur le site Web Les écrits de Harry Bernard, [http://harrybernard.cegepmontpetit.ca/reception-critique-des-oeuvres-de-harry-bernard/ juana-mon-aimee/] (20 mai 2015).

57 Ernest-Jules Larivière, "Un nouveau roman d'Harry Bernard ", Mon Magazine, novembre 1931, p. 2 et 34. 
de la psychologie féminine, conclut, malgré tout, que l'auteur est " resté vrai, vivant et captivant » et que Juana marque un progrès "bien accentué " par rapport aux romans précédents. Donatien Frémont, qui, rappelons-le, a commenté le roman, le résume plus qu'il ne le critique. Rédacteur de La Liberté $e^{58}$ au Manitoba, il retient que l'œuvre a le mérite "de faire connaître l'Ouest canadien ". Raphaëlle dans L'Action populaire ${ }^{59}$ trouve qu' i il y a de l'âme dans ce roman!».

Toutefois, un pavé remet en question ce bilan plutôt positif. Il est lancé par Lucien Parizeau dans La Patrie du 7 novembre $^{60}$. Sarcastique, ce dernier écrit :

M. Bernard a écrit, avec Juana, mon aimée, une de ses œuvres les plus lisibles. Son régionalisme y sent moins la bibliothèque que celui de ses autres ouvrages. [...] Les naïvetés y sont des perles que je n'ôterais pas si j'étais à la place de l'auteur. Ainsi je ne sais rien de plus gai que cette réflexion d'un « journaliste » sur l'argent, roi du monde : "Je n'avais jamais tant possédé de ce vil métal ». $\mathrm{Y}$ a une limite à tout, Harry.

Près d'un mois après sa sortie, le roman n'a reçu que les recensions présentées jusqu’à maintenant. Bernard a bien conscience que les critiques influents, à l'exception de Harvey, n'ont encore rien écrit à son sujet et qu'ils attendent de connaître quelle sera l'opinion dominante. Inquiet, il s'en ouvre non seulement à DesRochers ${ }^{61}$, mais également à Claude-Henri Grignon : "Les critiques vraies, hors Harvey, n'ont rien dit. On dirait que la plupart cherchent à prendre le vent ${ }^{62}$."

${ }^{58}$ Le Liseur (pseudonyme de Donatien Frémont), "Juana, mon aimée par Harry Bernard ", La Liberté, 4 novembre 1931, p. 3.

59 Raphaëlle (pseudonyme), "Juana, mon aimée, roman par Harry Bernard ", L'Action populaire, 5 novembre 1931.

${ }^{60}$ Lucien Parizeau, "Sur deux livres ", La Patrie, 7 novembre 1931, p. 17.

${ }^{61} \mathrm{HB}$ à $\mathrm{AD}, 6$ novembre 1931, Conversation poétique, p. 233.

${ }^{62} \mathrm{HB}$ à Claude-Henri Grignon, 16 novembre 1931, BAnQ-VM, Fonds HB, MSS298/011/001. 


\section{Un premier point tournant : l'entrée en scène de Grignon}

Suivant la recommandation de DesRochers, Bernard avait rendu visite à Grignon, à sa résidence de Sainte-Adèle, le 25 octobre $^{63}$. Quelques semaines plus tard, Grignon lui témoigne, dans une lettre, toute son admiration pour Juana et l'informe qu'il publiera prochainement un article en ce sens ${ }^{64}$. Cette lettre éclaire bien le rôle que va jouer Grignon, alias Valdombre, dans le système des critiques relatives au roman.

Mon cher Bernard,

Je viens de lire votre Juana qui est une sacrée belle chose. Je dois vous dire tout de suite sans phrases et sans génuflexions que c'est à peu près la seule œuvre importante et réellement artistique que vous ayez écrite. Je sais ce que je dis. Votre roman il marche en maudit, et là où les critiques distraits et pince-sans-rire n'ont vu que Juana et ses courses folles dans les prairies; moi, sans me vanter j'ai vu autre chose que ça. Aussi ai-je écrit un article sacrément exalté à ce sujet que je viens d'envoyer à Asselin ${ }^{65}$. J'ose espérer qu'il le publiera tel quel, malgré la botte formidable que j'applique avec force à M. Jean Béraud de LA PRESSE et à de certains "retours d'Europe ». J'y suis allé de mon humeur coutumière; et j'y trouve des idées qui vont ébranler les vieilles colonnes de notre abrutissement national. J'ai lu cet article à Pelletier ${ }^{66}$ qui l'a trouvé bien, et mieux peut-être. Il ne partageait pas d'abord

${ }^{63} \mathrm{HB}$ à AD, 27 octobre 1931, Conversation poétique, p. 222. Nous sommes portés à croire qu'au cours de cette visite Bernard lui aurait remis un exemplaire de son roman.

${ }^{64}$ Claude Henri Grignon à HB, 12 novembre 1931, BAnQ-VM, Fonds HB, MSS298/011/001. Ajoutons que le 16 novembre, Bernard l'informe, non sans arrière-pensée, que Dantin partage son enthousiasme (HB à Claude-Henri Grignon, BAnQ-VM, Fonds HB, MSS298/011/001).

${ }_{65}$ Olivar Asselin, rédacteur en chef du quotidien montréalais Le Canada, où Grignon publiera effectivement son article.

${ }^{66}$ Il s'agit ici d'Albert Pelletier, critique influent et redouté, et non de Georges Pelletier, rédacteur en chef du Devoir. Notaire de formation, il occupe le poste de registraire adjoint au Bureau d'enregistrement de la Ville de Montréal et collabore régulièrement à La Revue moderne et au quotidien Le Canada. 
mes vues, mais l'article paraît l'avoir convaincu car j'apporte des preuves à l'appui de mon argumentation en votre faveur.

Votre Juana m'étonne d'abord par la couleur locale et cette couleur est fournie par votre style imagé, rapide quand même qui la sert si victorieusement. Votre Lucienne est un rêve, comme écriture, et la compagne de Lebeau. C'est très beau et presque parfait en plusieurs endroits. Surtout le dialogue qui suit le retour de Madame Lebeau. C'est épatant. Quel naturel! Je ne viens pas vous flatter, Bernard. C'est contre mes habitudes. Mais je profite de votre livre pour vider bien des querelles et des différends ${ }^{67}$. Il était temps. N'allez pas croire surtout que votre visite a pu agir sur moi et faire pencher ma plume par sympathie. Rien de ça. Je persiste à dire que c'est le premier roman réel et réellement écrit que vous ayez écrit. Je dis même que c'est là le seul roman canayen, écrit par un Canayen. Pelletier n'a pas dit le contraire. J'attends les autres avec un fanal. Dans mon livre de critiques qui paraitra au cours de l'hiver, je reproduirai (mais avec plus de citations) l'article que je viens d'adresser à $\mathrm{M}$. Asselin qui se fera un plaisir et une justice de publier car il vous admire.

Vous n'êtes pas seulement un travailleur, vous êtes un créateur, et votre Juana est une sacrée belle fille, je vous le répète en vous tendant les mains,

Valdombre ${ }^{68}$.

Mentionnant à deux reprises la discussion qu'il a eue avec Pelletier, Grignon souligne de belle façon le rôle des propos informels entre certains critiques littéraires de l'époque. À son domicile rue Saint-Hubert, Pelletier invite ${ }^{69}$ les DesRochers, Grignon et autres

67 Grignon avait détesté les Essais critiques que Bernard avait publiés en 1929 et il ne s'était pas gêné pour le faire savoir dans une longue recension qu'il a reproduite dans son ouvrage Ombres et clameurs, Montréal, Albert Lévesque, 1933, p. 173-194 (voir le site Web Les écrits de Harry Bernard, [http://harry-bernard.cegepmontpetit.ca/ reception-critique-des-oeuvres-de-harry-bernard/essais-critiques/] (20 mai 2015)).

68 Claude Henri Grignon à HB, 12 novembre 1931, BAnQ-VM, Fonds HB, MSS298/011/001.

69 Voir Saint-Jacques et Robert, La vie littéraire au Québec, p. 171-172. 
pour s'entretenir de la valeur des œuvres littéraires du jour ${ }^{70}$. Non seulement Grignon et Pelletier ont-ils discuté du roman, mais la correspondance nous apprend que Pelletier et DesRochers, dès la fin d'octobre, en ont aussi débattu à leur tour : "Pelletier s'accorde à dire que ton Juana est ton meilleur livre, et de beaucoup, bien que la dédicace matamore le pique un peu au vif ${ }^{71}$. "Cela dit, on apprendra plus tard que Pelletier n'a pas beaucoup apprécié Juana. Les avis de DesRochers et de Grignon l'ont-ils incité à se taire provisoirement, lui qui pourtant s'était rapidement lancé dans la bataille en écorchant La ferme des pins, dans La Revue moderne dès janvier 1931 (le roman avait été mis en vente le 20 novembre ${ }^{72}$ ) et en faisant de même avec un autre roman de Bernard, Dolorès, sorti à la mi-novembre 1932, et qu'il égratigne à nouveau, six semaines plus $\operatorname{tard}^{73}$ ?

Il ne faut donc pas négliger ces discussions de salon, ces débats privés qui, à l'instar de la correspondance, jouent le rôle de médiateur dans les jugements littéraires. Grignon en est d'ailleurs fort conscient et quand il réunit, en 1933, ses critiques sous le titre Ombres et clameurs, il mentionne qu' " on parle tant de M. Bernard dans les journaux, dans les revues, dans les salons, partout du premier janvier au trente-et-un décembre ${ }^{74}$ ".

70 Au sujet de la tenue de ces rencontres informelles, voir les lettres envoyées par $\mathrm{AD}$ à HB, les 21 avril 1930, 16 septembre 1930 et 5 février 1931, Conversation poétique, p. 99, 125 et 178.

${ }^{71} \mathrm{AD}$ à HB, 27 octobre 1931, BAnQ-VM, Fonds HB, et Conversation poétique, p. 223. Cette dédicace que Pelletier a trouvé irritante se lit comme suit : «À toi Juana qui es et qui n'es pas qui aurais pu être et qui ne sera jamais. "

72 Albert Pelletier, "La ferme des pins par M. Harry Bernard ", La Revue moderne, $12^{\mathrm{e}}$ année, $\mathrm{n}^{\mathrm{o}} 3$ (janvier 1931), p. 10, voir le site Web Les écrits de Harry Bernard, [http://harry-bernard.cegepmontpetit.ca/reception-critique-des-oeuvres-de-harrybernard/17950-2/] (20 mai 2015).

73 Albert Pelletier, «Dolorès d'Harry Bernard », Le Canada, 27 décembre 1932, p. 2, voir le site Web Les écrits de Harry Bernard, [http://harry-bernard.cegepmontpetit. $\mathrm{ca} /$ reception-critique-des-oeuvres-de-harry-bernard/dolores/] (20 mai 2015). Une autre preuve de ce silence est qu'il continue, pendant ce temps, à publier des comptes rendus sur d'autres ouvrages, comme ceux qui paraissent dans Le Canada du 4 et du 22 décembre 1931, et qui portent, respectivement, sur Gloses critiques, de Louis Dantin, et sur Metropolitan Museum, de Robert Choquette.

74 Grignon, Ombres et clameurs, p. 174-175. 
Sur un autre plan, la lettre de Grignon livre fidèlement le contenu de son compte rendu, qui paraît finalement à la une du Canada le 18 novembre. On y trouve une phrase qui contribuera au succès de Juana en librairie : "Voilà un grand livre moral, lequel par la crise économique terrible que nous traversons, devrait être dans toutes les maisons, devrait être lu autour de la table familiale, pour le plus grand bien des ouvriers et des cultivateurs ${ }^{75}$."

À coup sûr, le sens du vent était donné. De ce jour jusqu'au prix David, ce sera le discours dominant ${ }^{76} \mathrm{du}$ système critique et ce n'est certes pas la remarque somme toute anodine d'Asselin, qui lui reproche d'avoir fait " plonger " les rats musqués, qui y changera quoi que ce soit $^{77}$.

Profitant de la sortie de presse matinale du Canada, Parizeau réagit le jour même ${ }^{78}$ à la critique que lui a subtilement adressée Grignon dans son compte rendu. En fait, non seulement Grignon y tenait des propos dithyrambiques sur Juana, mais il prenait la défense de l'œuvre en s'attaquant à ceux qui l'avaient critiquée. Et sa charge a eu de l'effet, puisque Parizeau met maintenant de l'eau dans son vin, tout en cherchant à ne pas perdre la face :

Que M. Bernard ait écrit, avec Juana, mon aimée, son meilleur roman, il ne se trouvera personne pour le nier; et cet excellent Valdombre se bat contre des moulins quand il promène sa colère au nez des lecteurs. La sagesse lui conseillerait d'enfourcher, de

75 Valdombre, "Le dernier roman de M. Harry Bernard, Juana mon aimée ", Le Canada, 18 novembre 1931, p. 9. Mentionnons que sa recension sera reprise dans La Survivance, le 16 décembre 1931.

76 Voir, à propos de l'importance du discours dominant dans la réception critique, Chartier, L'émergence des classiques, p. 29-31.

77 Olivar Asselin, "À propos de rat musqué ", Le Canada, 18 novembre 1931, p. 1. Selon Asselin, Bernard aurait fait une erreur en décrivant les rats musqués qui plongent dans l'eau, ce qui avait amené Grignon, piqué par cette remarque disgracieuse d'Asselin, à s'en enquérir auprès de Bernard (voir Claude-Henri Grignon à HB, 20 novembre 1931, et $\mathrm{HB}$ à Claude-Henri Grignon, 25 novembre 1931, BAnQ-VM, Fonds HB, MSS298/011/001).

78 Lucien Parizeau, "Une prière : à un critique sans pitié ", La Patrie, 18 novembre 1931, p. 17. 
temps en temps, l'âne de Panco. Il tempête, à tort, contre cette phrase que j'ai écrite (je sais qu'il s'adresse à votre serviteur) : «M. Bernard a écrit son œuvre la plus lisible ". Si mon ami n'avait point perdu le sens des mots il saurait peut-être que lisible n'est pas péjoratif. Je me soucie peu d'être français; mais je voudrais encore moins emprunter à Cicéron ou aux chargés de publicité la manie des superlatifs. C'est rendre un mauvais service à M. Bernard que de l'inviter, même en esprit, à un concubinage gourmand avec les chefs-d'œuvre. Tout le monde qui sait lire félicitera l'auteur de Juana d'avoir écrit, non une œuvre excellente par la vérité de l'inspiration et la correction de la langue, mais un roman plus intéressant que tous les autres romans de notre littérature, à l'exception de la Pension Leblanc.

Dès le 20 novembre, Grignon réécrit à Bernard afin de lui souligner la belle publicité que ses échanges avec Parizeau ont provoquée. "Vous devez être heureux : la bataille est engagée autour de votre Juana, et ces coups de sabre et ces décharges de balles vont vous faire une publicité épatante ${ }^{79}$. " Conscient que Pelletier n'a encore rien publié, Grignon souhaite le voir entrer dans la bataille. "Tant mieux. C'est ce que je souhaite », ajoute-il, « et ne céderai pas d'un pouce ${ }^{80}$. " Mais Pelletier reste silencieux encore dix mois.

De son côté, Bernard s'active à promouvoir son œuvre. Au début de novembre, il inscrit son roman au prix d'Action intellectuelle. Le 4, il le soumet aux éditions Bernard Grasset afin de lancer une édition en France ${ }^{81}$. Mais, le 18 novembre, l'éditeur refuse : "Il est de règle stricte pour nous de ne jamais réimprimer un livre qui a déjà été mis en vente ${ }^{82}$. » Le même jour, il enregistre ses droits d'auteur à Ottawa. Deux jours plus tard, il reproduit de larges extraits de la recension

\footnotetext{
79 Claude-Henri Grignon à HB, 20 novembre 1931, BAnQ-VM, Fonds HB, MSS298/011/001.

80 Ibid.

81 HB à Bernard Grasset, 4 novembre 1931, BAnQ-VM, Fonds HB, MSS298/011/001.

82 Pierre Tisne à HB, 18 novembre 1931, BAnQ-VM, Fonds HB, MSS298/011/001.
} 
de Valdombre dans Le Courrier, avec en sous-titre « Une magnifique appréciation critique de Valdombre ${ }^{83}$ ".

Ayant reçu de Joseph-Marie Melançon, alias Lucien Rainier, une lettre le félicitant de son roman Juana, malgré quelques tournures grammaticales incorrectes ${ }^{84}$, Bernard profite de l'occasion pour lui mentionner, le lendemain 23 novembre : "Cela ne me fâche pas qu'on m'indique mes fautes. Au contraire, j'essaie toujours de faire mon profit des critiques qui me sont adressées. Ainsi, dans les circonstances, je vous serais reconnaissant de bien me préciser ce que vous m'indiquez dans votre lettre ${ }^{85}$. " Même si le livre se vend bien ${ }^{86}$, Bernard n'envisage pas encore une réimpression, mais souhaite corriger ses erreurs " dans une autre édition, - si jamais, $-\ldots{ }^{87}$ ». Le 26 novembre, Rainier lui transmet ses notes comportant une vingtaine de corrections et termine sa lettre avec humour par la phrase suivante : "ALLEZ EN PAIX ET NE PÉCHEZ PLUS ${ }^{88}$. " Et il faut attendre la réimpression du roman, le 25 mai 1932, pour constater que l'auteur a tenu compte de presque toutes les remarques de Rainier.

Grignon n'a pas terminé son travail de promotion de Juana. Sont-ce les conversations avec Pelletier ou le compte rendu fort mitigé,

${ }^{83}$ Cette deuxième version, qui n'avait pas besoin d'être expurgée de ses passages négatifs, est très semblable à la première.

${ }^{84}$ Joseph-Marie Melançon à HB, 22 novembre 1931, BAnQ-VM, Fonds HB, MSS298/011/001.

85 HB à Joseph-Marie Melançon, 23 novembre 1931, BAnQ-VM, Fonds Sœur Marie-Henriette-de-Jésus, MSS17/03. C'est grâce aux transcriptions d'une partie de la correspondance de Melançon par sœur Marie-Henriette-de-Jésus que nous avons pris connaissance de cette lettre. Voir aussi sœur Marie-Henriette-de-Jésus, Un ami intime de Nelligan, Lucien Rainier (abbé Joseph-Marie Melançon) : l'homme et l'œuvre, Montréal, Éditions du Lévrier, 1966.

86 Selon un communiqué publié par Le Bien public en 1947 à la suite d'une nouvelle édition de Juana, l'édition originale de 1931 se serait écoulée en deux mois, ce qui nous paraît bien exagéré ("Juana, mon aimée par Harry Bernard », 17 avril 1947, p. 5).

${ }^{87}$ HB à Joseph-Marie Melançon, 23 novembre 1931, BAnQ-VM, Fonds Sœur Marie-Henriette-de-Jésus, MSS17/03.

${ }^{88}$ Joseph-Marie Melançon à HB, 26 novembre 1931, BAnQ-VM, Fonds HB, MSS298/011/001. 
paru dans la revue des étudiants de l'Université de Montréal, Le Quartier latin, à la fin de novembre, qui l'incitent à remonter aux barricades ${ }^{89}$ ? Nous l'ignorons. Empruntant un autre pseudonyme, Des Esseintes, il fait paraître, au début de janvier 1932, un autre long texte dithyrambique dans La Revue populaire, dans lequel il se cite lui-même en empruntant un passage à Valdombre : "Juana mon aimée est une envoûtante figure et elle ne déparerait point la galerie maintenant célèbre où domine Maria Chapdelaine $e^{90}$. " Bernard, bien évidemment, s'empresse de reproduire l'article le 8 janvier dans $L e$ Courrier, sous le titre "Le beau succès de Juana, mon aimée ${ }^{11}$ ".

Par la suite, au cours des premiers mois de 1932, paraissent au moins huit comptes rendus favorables. Les Hébert ${ }^{92}$, Bruchési ${ }^{93}$, Dantin $^{94}$, Roy ${ }^{95}$ et Thomas-Marie Lamarche ${ }^{96}$ y ajoutent leur grain

89 Vigilantia (pseudonyme), "Juana, mon aimée », Le Quartier latin, 26 novembre 1931, p. 6. En tout état de cause, ce n'est certainement pas le seul compte rendu négatif publié en novembre qui aurait pu l'inciter à reprendre du collier, car celui que signe Georges-Émile Marquis dans Le Terroir reste fort positif, tout comme celui que Frémont reprend et développe dans La Liberté du 28 novembre.

90 Des Esseintes (pseudonyme de Claude-Henri Grignon), "Juana, mon aimée, roman par Harry Bernard ", La Revue populaire, janvier 1932, p. 50.

91 Des Esseintes, "Le beau succès de Juana, mon aimée ", Le Courrier de SaintHyacinthe, 8 janvier 1932, p. 1.

92 Maurice Hébert, "Au tournant romanesque de nos lettres ", Le Canada français, vol. 19, no 5 (janvier 1932), p. 371-383.

93 Jean Bruchési, "Dans le monde des lettres : trois romans ", La Revue moderne, $13^{\mathrm{e}}$ année, $\mathrm{n}^{\circ} 4$ (février 1932), p. 16-17. Bernard le remercie d'ailleurs de cette recension le 3 février (HB à Jean Bruchési, BAnQ-VM, Fonds HB, MSS298/044/005).

${ }^{4}$ Louis Dantin (pseudonyme d'Eugène Seers), "Juana, mon aimée, roman de M. Harry Bernard ", L'Avenir du Nord, 26 février 1932, p. 1 et 2.

95 Camille Roy, "Juana, mon aimée ", L'Enseignement secondaire au Canada, mars 1932, p. 456-463.

96 Thomas-Marie Lamarche, "Harry Bernard : Juana, mon aimée ", Revue dominicaine, février 1932, p. 124-125. On notera que le responsable de cette publication, son homonyme, le père Marc-Antonin Lamarche, s'est excusé auprès de Bernard de la parution tardive de cette recension, ce qui indique une certaine complicité entre eux (Marc-Antonin Lamarche à HB, 7 janvier 1932, BAnQ-VM, Fonds HB, MSS298/011/001). 
de sel. Même Robert Rumilly y trouve quelques qualités ${ }^{97}$. Comme l'indique Dantin le 26 février :

Tout semble avoir été dit sur ce livre, et avec un accord qu'atteint rarement la critique. Quand on voit opiner du même bonnet $\mathrm{M}$. Jean Bruchési et $\mathrm{M}$. Claude Grignon, et des jugements identiques jaillir de cerveaux si divers, on peut se dire que la vérité objective est enfin sortie de son puits et s'est montrée splendidement nue ${ }^{98}$.

\section{En route vers le prix David}

Tout en écrivant un nouveau roman, Dolorès, dont Séraphin Marion dira plus tard qu'elle est la sœur jumelle de Juana ${ }^{99}$, Bernard accumule les bonnes nouvelles. À la fin d'avril 1932, il remporte un prix d'Action intellectuelle dans la section littérature pour Juana ${ }^{100}$. C'est le sixième prix que lui décerne l'Association catholique de la jeunesse canadienne-française, et la somme versée de 100 \$ est une gracieuseté du journal La Presse. Sans doute espère-t-il remporter un prix David dont la date de clôture des mises en candidature est le $1^{\text {er }}$ mai ${ }^{101}$ et où sont inscrits, depuis janvier, ses deux derniers romans ${ }^{102}$.

Ayant épuisé son inventaire et devant le succès critique du roman, Lévesque décide, en mai, de lancer une nouvelle édition et imprime donc 1000 autres exemplaires aux presses du Courrier. Si l'on en juge par le nombre de livres publiés cette année-là, le monde de l'édition québécoise, malgré la crise économique, ne se porte pas aussi

97 Robert Rumilly, «La vie littéraire : Juana, mon aimée ", Le Petit Journal, 7 février 1932, p. 4. Bernard avait été prévenu par Rumilly de la publication prochaine de cette recension dans une lettre du 17 janvier 1932 (Robert Rumilly à HB, BAnQ-VM, Fonds HB, MSS298/047/011).

98 Louis Dantin, "Juana, mon aimée : roman de M. Harry Bernard ", L'Avenir du Nord, 26 février 1932, p. 1.

99 Voir Séraphin Marion, "Deux nouveaux romans ", Revue de l'Université d'Ottawa, avril-juin 1933, p. 229-244. Malgré le titre, Marion s'intéresse en fait dans ce compte rendu à trois livres, soit Juana, Dolorès et Cap Blomidon.

100 "Les lauréats des prix d'Action intellectuelle », Le Devoir, 28 avril 1932.

101 Albert Lévesque, "Le prix David (1930-31) ", Almanach de la langue française 1932, Montréal, Albert Lévesque, 1932, p. 251.

102 HB à Albert Lévesque, 15 janvier 1932, BAnQ-VM, Fonds HB, MSS298/046/014. 
mal qu'on aurait pu le penser. Comme le soulignent Saint-Jacques et Robert, en ce qui a trait à la production littéraire, "les effets de la crise économique se font finalement sentir [...] après $1933^{103}{ }$. Non seulement on réédite le roman, mais Bernard, sans doute avec l'aval de Lévesque, autorise, à partir du mois de mai, sa reproduction intégrale dans Mon Magazine ${ }^{104}$. Toutefois, les difficultés financières de la revue interrompent le projet; le numéro de juillet, qui aurait dû contenir le dernier tiers du roman, ne sera jamais publié.

Le succès de Juana est tel que La Presse, peut-être informée de la mésaventure de Mon Magazine, demande à Bernard, le 14 juin ${ }^{105}$, son autorisation pour insérer Juana en feuilleton dans le quotidien. Compte tenu de la publicité ainsi apportée au roman, le journal estime inutile le versement d'une compensation financière.

Il nous souvient que MARIA CHAPDELAINE de Louis Hémon, provoqua un immense intérêt au Canada, du jour où La Presse le publia dans des conditions à peu près semblables à celles que je vous propose pour votre ouvrage. Bernard Grasset, propriétaire des droits d'auteur de MARIA CHAPDELAINE, consentit volontiers à nous laisser publier gratuitement le chef-d'œuvre de Louis Hémon, en considération de ce que lui vaudrait la publicité de La Presse.

Mécontent de l'offre, Bernard refuse la proposition : " Je proteste de toutes mes forces contre votre manière de faire, qui est une véritable exploitation de l'écrivain canadien ${ }^{106}$. "Selon lui, c'est une question

103 Saint-Jacques et Robert, La vie littéraire au Québec, p. 221-222.

104 Cela s'explique peut-être par le fait qu'un des artisans de cette revue est JulesErnest Larivière, critique littéraire et connaissance de longue date de Bernard. Comme Larivière l'écrivait lui-même en janvier 1931 à propos de La ferme des pins, « [j]e voudrais n'avoir que du bien à dire du nouveau roman de Bernard [...] parce que l'ami Harry est un brave maskoutain que j'ai connu bambin » ("La ferme des pins par Harry Bernard ", Mon Magazine, p. 3; voir aussi le site Web Les écrits de Harry Bernard, [http://harry-bernard.cegepmontpetit.ca/ reception-critique-des-oeuvres-de-harry-bernard/17950-2/] (20 mai 2015)).

105 Oswald Mayrand à HB, 14 juin 1932, BAnQ-VM, Fonds HB, MSS298/011/002.

106 HB à Oswald Mayrand, 22 juin 1932, BAnQ-VM, Fonds HB, MSS298/011/002. 
de principe ${ }^{107}$, bien plus qu'une question d'argent, car il demande habituellement 25 \$ pour la publication en feuilleton d'une de ses œuvres ${ }^{108}$.

Tout en veillant de près au sort de Juana, Bernard milite activement dans la section française de l'Association des auteurs canadiens afin de promouvoir la vente de livres canadiens. En février, il a proposé à Jean Bruchési, président de l'Association, la tenue d'une campagne de presse en faveur de l'achat de livres de récompense canadiens par les commissions scolaires. L'objectif est d'exiger le respect intégral de la loi Choquette, adoptée en 1925, qui oblige, en principe, les commissions scolaires à consacrer la moitié de leur budget à l'achat de livres d'ici ${ }^{109}$. Cette loi, appliquée en partie, aurait eu, malgré tout, des effets déterminants sur le développement de l'institution littéraire québécoise ${ }^{110}$.

Avec l'appui de Bruchési ${ }^{111}$, Bernard lance cette campagne le $1^{\text {er }}$ avril en publiant dans Le Courrier un premier éditorial consacré à cette question. En deux mois, il publie sur le sujet six articles en

107 Finalement, ce n'est qu'un an plus tard que Bernard va consentir à faire paraître Juana sous forme de feuilleton. Le Bien public l'offrira à ses lecteurs du 23 mars au 8 juin 1933, officiellement après avoir versé $25 \$$, alors que dans les faits Bernard avait exempté le journal de ce paiement.

108 Comme il le précise à Clément Marchand du Bien public dans une lettre du 17 mars 1933. On pourra lire une bonne partie de la correspondance échangée entre Marchand et Bernard sur le site Web Les écrits de Harry Bernard, voir la lettre 16, [http://harry-bernard.cegepmontpetit.ca/correspondance/la-correspondanceentre-harry-bernard-et-clement-marchand] (19 mars 2016).

109 HB à Jean Bruchési, 10 février 1932, BAnQ-VM, Fonds HB, MSS298/044/005. Bernard écrit : "Nous pourrions nous combiner, plusieurs journaux et revues, pour lancer le cri. Ce serait le temps, vu que les achats de livres de récompenses, dans les commissions scolaires, se font à partir d'avril. Si ce projet vous intéresse, je suis prêt à attacher le grelot. J'écris à DesRochers, Haines, Harvey, Barrette du Droit, etc., leur demandant de suivre. La Revue moderne se mettrait de la partie, et sans doute le Populaire, Mon magazine, etc. Il y aurait moyen, je crois, de faire un beau chahut. "

110 Saint-Jacques et Robert, La vie littéraire au Québec, p. 234.

111 Jean Bruchési à HB, 12 février 1932, BAnQ-VM, Fonds HB, MSS298/044/005. Tout en approuvant cette campagne, Bruchési lui demande de retarder quelque peu son lancement en raison d'un calendrier politique défavorable. 
Figure 1

Une publicité annonçant l'obtention du prix David parue dans Le Courrier de Saint-Hyacinthe du 4 novembre 1932 au 27 janvier 1933

le livre de l'année

Juana, mon aimée $\% M+\pi$ par HARRY BERNARI)

\section{grand prix David, 1932 prix d'action intellectuelle}

En vente au Courrier de Saint-Hyacinthe $\$ 1.00$ l'ex

première page, dont trois éditoriaux. La campagne, à laquelle se joignent effectivement d'autres journaux, aura de nombreuses répercussions et de belles retombées, si l'on en croit Lévesque qui, dans une lettre circulaire, envoyée notamment à Bernard, indique que la démarche a permis à plusieurs livres canadiens d'atteindre une deuxième édition ${ }^{112}$.

\section{Un autre point tournant : l'intervention de Pelletier}

Devant le concert de louanges adressées à Juana, Pelletier décide de retenir sa critique jusqu'au dévoilement des prix David le 19 septembre. Cette critique percutante, s'il en est une, paraît finalement trois jours après le dévoilement du nom des lauréats, dans Le Bien

112 Albert Lévesque à HB, 13 juillet 1932, BAnQ-VM, Fonds HB, MSS298/044/005. 
public. Un texte de présentation ${ }^{113}$, signé Clément Marchand, précède la recension.

La critique en général fut laudative pour ce dernier-né de M. Bernard. En raison, «Juana, mon aimée » est une réalisation. Cependant M. Albert Pelletier, auteur de Carquois, a sourcillé malicieusement avant de se découvrir. Il nous communiqua en août une magistrale analyse de " Juana " avec note de ne la publier qu'après l'attribution du Prix David. Ce qui prouve que toute idée de nuire est absente de lui. La présente critique s'avère sarcastique, violente mais " RAISONNABLE " par-dessus tout (Albert Pelletier a vécu dans l'Ouest canadien). De nos romanciers canadiens seul Harry Bernard, croyons-nous, est assez fort pour résister à de tels coups de bélier, sans cracher le sang ${ }^{114}$.

Dans ce texte sarcastique et dévastateur, Pelletier confirme, dès la première ligne, le rôle clé joué jusqu'alors par Grignon. Afin de rendre compte de l'ampleur et de la dureté de ses remarques - qui insistent longuement sur les invraisemblances des situations et des attitudes des personnages et sur la méconnaissance des plaines de l'Ouest canadien -, citons-en quelques extraits :

Quand Valdombre veut rendre un service, il a au moins assez de talent pour réussir. Le grelot qu'il attacha à Juana, " mon aimée " entraîna tout le troupeau de nos critiques de bergerie. [...] Tous [...] arrivent à Juana les yeux accrochés aux étoiles et le crâne éclatant d'admiration. C'est que ce grand lyrique, Valdombre, leur a insufflé l'extase! Il les a tous hypnotisés!

\section{$[\ldots]$}

La Saskatchewan $\left[c^{\prime}\right]$ est $[\ldots]$ presque aussi loin que la lune, et voilà pourquoi Juana, mon aimée est un si beau roman. Malheureusement, je connais la Saskatchewan de ce temps-là.

113 La présence de deux erreurs dans ce texte (le nombre de prix David remportés par Bernard et la date de parution de Juana) indique clairement que Bernard n'a pas été consulté sur cette démarche.

114 Clément Marchand, "Juana, mon aimée, d'Harry Bernard : note de la rédaction ", Le Bien public, 22 septembre 1932, p. 3. 
[...] Juana perd beaucoup de son charme et me paraît un livre bien inférieur au précédent roman d'Harry Bernard, La ferme des pins.

$[\ldots]$

[Bernard] déclare que tout le monde s'est trompé, que « la plaine est extrêmement diverse ». (Et il ne note même pas, la littérature gouvernementale n'en faisant pas mention, les rouges, hauts, bien visibles greniers des coopératives qui balisent partout les voies ferrées!) Mais il est encore plus fantaisiste. "Des lacs nombreux apparaissent, vert-bleu ou gris d'argent, dans le lointain... " Mon cher Bernard, ce ne sont pas des lacs que vous avez vus, c'est le mirage, phénomène bien remarquable dans la Prairie, dont vous ne dites pas un mot!

$$
[\ldots]
$$

Je résume mes impressions. Harry Bernard possède un grand défaut d'observation et de style. Qu'il s'agisse de caractères, d'une intrigue, d'un milieu, il n'en aperçoit pas l'ensemble ni, c'est entendu, l'apparence artistique, l'effet total. De là ces invraisemblances des personnes et de leur histoire, ces généralisations ou ces morcellements, outrés jusqu’à la destruction de la vérité, des lieux où on les fait vivre. De là aussi cette maigreur générale du style. La sensibilité et l'imagination de l'auteur s'avèrent myopes. [...] De plus, parce qu'il ne voit que par alinéas ou par pages, il ne remarque pas que l'ensemble du récit manque de chaleur, que Raymond Chatel se montre vraiment trop à court de lyrisme. Défaut d'autant plus remarquable, pourtant, qu'il s'agit d'un roman d'amour, et que ce roman est écrit à la première personne ${ }^{115}$.

En taillant en pièces les arguments des partisans de Juana, obnubilés par le caractère exotique de la Saskatchewan, et en calculant bien sa sortie, Pelletier va rapidement imposer un nouveau point de vue qui, depuis, est resté sans appel. Ainsi, deux semaines plus tard, son article est repris intégralement dans La Revue de Granby, accompagné d'un titre plus qu'évocateur « Un mauvais quart d'heure

115 Albert Pelletier, "Juana, mon aimée d'Harry Bernard ", Le Bien public, 22 septembre 1932, p. 3 et 5 . 
pour Juana, mon aimée ${ }^{116}{ }^{\prime}$. Collaborateur régulier au Canada, Pelletier va également y publier sa critique de Juana à la fin de novembre ${ }^{117}$. Un nouveau discours dominant allait dorénavant s'installer.

Prévenu de sa parution, Bernard prend connaissance sans surprise de cette critique dévastatrice. En effet, Marchand, dans une lettre du 8 septembre, lui mentionne qu'il a tenté, en vain, de convaincre Pelletier de changer d'idée. "J'ai rendu visite à Pelletier. Il m’a lu sa critique sur Juana. Il n'est pas permis de lui ôter sa manière de voir. [...] Il a gardé sa copie. Il ne veut pas la publier avant l'attribution du prix David. Crainte de t'ôter des chances. Pouf! Je te ferai tenir une copie de cette critique avant de la publier, si toutefois je m'y décide $^{118}$.»

On ne connaît pas la réaction de Bernard après l'intervention de Pelletier : la correspondance qu'il entretient avec DesRochers se fait plus rare à ce moment-là tandis que celle avec Marchand reste muette sur le sujet ${ }^{119}$. Une lettre de Harvey au début d'octobre, soit après la publication de la critique de Pelletier, n'y fait pas davantage allusion. Membre du jury du prix David, Harvey le félicite et lui fait une confidence intéressante au sujet des discussions qui se sont déroulées entre les membres du jury du prix David :

Mon cher ami,

Inutile de te dire que j'étais heureux de contribuer à te donner une récompense pour ton travail, surtout pour ton progrès.

La discussion a porté entre toi et Desrosiers (Nord-Sud). Il manquait à ce dernier ce que tu possèdes : l'action ${ }^{120}$.

116 La Revue de Granby, 6 octobre 1932.

117 Le Canada, 25 novembre 1932.

118 Clément Marchand à HB, 8 septembre 1932, BAnQ-VM, Fonds HB, MSS298/011/002.

119 "Tu me diras ", écrit Marchand le 28 septembre, " ce que la critique d'Albert Pelletier t'a causé d'émotions " (Clément Marchand à HB, 28 septembre 1932, BAnQ-VM, Fonds HB, MSS298/046/020).

120 Jean-Charles Harvey à HB, 5 octobre 1932, BAnQ-VM, Fonds HB, MSS298/011/002. 
Le sort que connaitra le roman par la suite est beaucoup moins reluisant. En effet, deux critiques paraissent en 1933. La première, de Parizeau, revient sur la recension de Juana faite par Grignon, que ce dernier a reproduite dans Ombres et clameurs. "Mais quelque admiration que j'éprouve pour le lyrisme impérieux de Grignon, quelques-uns de ses jugements critiques me dépassent. Son éloge de Juana, mon aimée serait une embardée d'aveugle si ce n'était en vérité la poésie qu'un excessif a jetée dans un ouvrage médiocre dont les personnages vivent en automates, suivant un déterminisme arbitraire $^{121}$. " L'autre, signée K.C.K, paraît en anglais dans Books Abroad et se contente de ce commentaire : "too slight to be exciting ${ }^{122}$ ".

Que dire de Lévesque qui, en 1934, dénigre Juana? En présentant succinctement les romans de Bernard, dans son Almanach de la langue française, il fait maintenant sienne l'évaluation de Pelletier : "Avec ses deux derniers romans [Juana et Dolorès], il s'est essayé dans le roman psychologique. Mais là où il réussit le mieux, c'est dans la peinture de l'habitant canadien, et La ferme des pins est considéré à ce point de vue, comme son meilleur roman ${ }^{123}$. » Finalement, quand le roman est réédité en 1947, quelques autres recensions paraissent sans être très favorables ${ }^{124}$.

\section{$\sec 0$}

Il convient maintenant de tirer quelques observations du parcours du roman. Se confiant à Louis Dantin, DesRochers disait à propos de Bernard : "Il commence simplement à s'apercevoir que l'affaire du romancier, c'est de conter une histoire intéressante et non pas de

121 Lucien Parizeau, "Ombres et clameurs, discours critiques de Claude-Henri Grignon ", Le Canada, 18 mai 1933, p. 2.

122 K.C.K, "Harry Bernard, Juana mon aimée», Books Abroad, vol. VII (1933), p. 58.

123 Albert Lévesque, Almanach de la langue française, Montréal, Éditions Albert Lévesque, 1934, p. 70.

124 Voir, par exemple, Émile Bégin, « Notes de lecture : Juana, mon aimée ", L'Enseignement secondaire au Canada, vol. 27, n' 2 (décembre 1947), p. 145-146; Berthelot Brunet, "Monsieur Harry Bernard et son androgyne ", Le Canada français, 14 octobre 1947, p. 5. 
faire le travail de vicaire ${ }^{125}$. " Juana marque assurément un point tournant dans la carrière du romancier, qui s'éloigne enfin des romans à thèse.

Le principal intérêt du roman réside peut-être dans sa narration au JE et le monologue intérieur, qui permet l'abandon du narrateur omniscient. En proposant cette structure narrative, encore peu connue au Canada français, Bernard donne un peu d'air au champ littéraire sclérosé par les romans didactiques. En ce sens, Juana marque une étape dans l'évolution de la littérature d'ici ${ }^{126}$. Sans qu'on puisse l'associer aux deux courants qui commencent à transformer le champ littéraire au début des années $1930^{127}$, soit les romans de la « jeune génération " publiés par Lévesque et le renouvellement régionaliste, Juana participe à sa manière à cette évolution.

Toutefois, force est d'admettre la place marginale consacrée à ce procédé narratif dans les recensions, de sorte que l'obtention du prix David ne lui en serait nullement redevable. En effet, plusieurs auteurs, les Parizeau, Lamarche, Frémont, Rumilly, n'en font même pas mention, et quand le sujet est abordé, ce n'est que fort brièvement, comme chez Pelletier. Si le procédé a déplu à Hains (« Ah cette manie de monologue intérieur, ce qu'elle peut être rosse parfois!»), et si Hébert demeure dubitatif en le décrivant comme une " technique moderne, mais réfléchie ", il a plu à Grignon, qui le présente comme un procédé "que la plupart des romanciers modernes ont accepté ". Mal compris, le procédé en déroute quelques-uns. Ainsi, Camille Roy hésite à croire que le récit soit purement fictif: c'est « une histoire qui ne lui est peut-être pas arrivée ».

Un roman n'est jamais l'œuvre d'une seule personne. Mentors et amis y participent plus ou moins discrètement et mettent l'épaule à la roue à des degrés divers. Alfred DesRochers a joué un rôle clé dans le succès de Juana, et cela à plus d'un titre. Non seulement

\footnotetext{
125 Alfred DesRochers à Louis Dantin, 16 novembre 1931, Émulation littéraire, p. 385.

126 Jean-Paul Lamy, "Juana, mon aimée, roman de Harry Bernard ", dans Lemire (dir.), Dictionnaire des ceuvres littéraires du Québec, t. II : 1900 à 1939, p. 615.

127 Voir Saint-Jacques et Robert, La vie littéraire au Québec, p. 398-402.
} 
commente-t-il à deux reprises le manuscrit, mais il est à l'origine de la visite décisive - et sans doute calculée - que fait Bernard à Grignon. C'est encore lui, sans doute, qui mentionne le nom de Rainier, avec qui il correspond assidûment depuis des années et dont il apprécie la sincérité des remarques ${ }^{128}$. Il a aussi défendu le roman dans les salons où Bernard brille par son absence. Ces salons sont, dans le domaine privé tout autant que la correspondance, des lieux où se développe la critique. Outre le poète de Sherbrooke, il faut mentionner l'intervention non mesurable de Claude Melançon et celle, limitée, de Groulx et de Frémont.

Comme le montre la correspondance de Bernard, le domaine privé du système critique reste indissociable des recensions publiées même si Juana, à l'instar des autres romans de cette époque, fait l'objet de nombreux comptes rendus signés par presque tous les principaux ténors de l'époque. À ce chapitre, retenons l'influence de Grignon, qui a insuffé une direction à la critique, comme il le fera d'ailleurs pour Menaud, maître-draveur ${ }^{129}$. Pendant dix mois, son point de vue s'impose comme le discours dominant sur l'œuvre au point peut-être de faire taire temporairement Pelletier.

On le sait, un prix David n'est pas nécessairement une garantie de passer à l'histoire, et Juana en constitue une belle preuve. Malgré ses qualités, son côté novateur et tous les efforts de Bernard pour faire connaitre son roman, Juana a disparu des livres d'histoire littéraire. On peut penser que les raisons réelles de cette gloire éphémère sont à chercher du côté de l'intervention calculée et percutante de Pelletier. Reprise par deux journaux et incontestée par la suite, sa critique semble avoir joué un rôle décisif au point de devenir LE discours dominant sur l'œuvre. Comment " résister à de tels coups de bélier, sans cracher le sang ", écrivait Marchand. Chartier mentionnait ceci à propos des œuvres qui sont parvenues à faire partie de

\footnotetext{
128 Commentaire personnel que nous a livré Richard Giguère lors d'une conversation téléphonique le 5 janvier 2015.

129 Chartier, L'émergence des classiques, p. 119.
} 
l'histoire littéraire : "Souvent, un phénomène particulier, parfois un seul texte [...] détermine l'organisation du système de réception et favorise ou empêche la synthèse des opinions énoncées sur l'œuvre ${ }^{130}$. " Pelletier, non sans raison, aurait beaucoup contribué à écarter Juana des œuvres marquantes des années 1930. Il n’y a qu'à comparer les jugements portés sur l'œuvre avant et après son intervention pour s'en convaincre.

En résumant les années 1919 à 1933, Denis Saint-Jacques et Lucie Robert, dans leur ouvrage consacré à la vie littéraire, laissaient le lecteur sur ces mots, qui nous permettent de prendre un peu de recul :

Ces années d'après-guerre apparaissent au Canada français comme un moment décisif dans la spécification nationale étendue du champ littéraire. Cette évolution se réalise en une difficile conjonction avec le mouvement qui y fit reconnaître la voix individuelle des créateurs, celui de l'autonomisation littéraire proprement dite. Enfin, une telle confrontation se livre sur le marché littéraire, lui-même en voie de se définir comme un secteur relativement distinct. Trois acteurs illustrent cette intrication des champs, Groulx, l'idéologue, Dantin, l'esthète et Lévesque, l'éditeur : le nationaliste, l'individualiste et le marchand. Le nationaliste emploie d'abord le marchand pour assurer la diffusion de son idéologie, mais celui-ci, cherchant à diversifier sa production, en vient à offrir à l'individualiste et aux siens un lieu de publication. En bref, Lévesque, éditeur de Groulx, assure la fortune de Dantin, l'opposant de Groulx ${ }^{131}$.

Ces trois courants, qui traversent l'institution littéraire, ont assurément été personnifiés par Bernard. Ainsi, après avoir prêté sa plume à une littérature au service de la nation en récoltant deux prix David, il fait entendre avec Juana une voix novatrice qui séduit plusieurs critiques, tout en se montrant parallèlement préoccupé par la diffusion du livre canadien, comme le prouve sa lutte intéressée

130 Ibid., p. 30.

131 Saint-Jacques et Robert, La vie littéraire au Québec, p. 519-520. 
en faveur des livres de récompense. Somme toute, le travailleur méthodique que fut Harry Bernard était de son temps.

Mais pour passer à l'histoire, peut-être aurait-il fallu qu'il ne soit pas de son temps! Non seulement ce roman a été effacé de l'histoire littéraire, mais c'est toute son œuvre, inscrite dans quatre champs de l'histoire culturelle, qui a subi le même sort. En effet, outre le romancier, il y a le naturaliste Bernard qui a rédigé de nombreux articles sur la faune et la flore, sans compter ce bel essai de vulgarisation scientifique que constitua l' $A B C$ du petit naturaliste canadien. Parallèlement, en tant que critique littéraire, il a fait paraître, sous le nom de plume de l'Illettré, plus de 1500 critiques en une trentaine d'années. Que dire maintenant du journaliste et du rédacteur en chef qui, en un demi-siècle, a publié plus de 2000 éditoriaux, a été membre fondateur de l'Association des hebdomadaires en 1932 et, en 1933, le premier rédacteur en chef de L'Action nationale? Les motifs de son effacement de la mémoire collective, qu'il faudra peut-être chercher ailleurs que dans la seule institution littéraire, demeurent encore obscurs et constituent certainement un défi à relever. 